Article

\title{
Novel Approaches to Chemically Cross-linked Phosphazene Structures: Hydrosilylation vs Piers- Rubinsztajn reaction
}

\author{
Andrey S. Esin, Anna I. Chernysheva, Mikhail A. Soldatov, Ekaterina A. Karpova, Vyacheslav V. \\ Kireev, Igor S. Sirotin, Mikhail V. Gorlov* \\ Mendeleev University of Chemical Technology of Russia, 125047, Miusskaya sq. 9, Moscow 125047, Russia \\ * Correspondence: mikgorlov@gmail.com
}

\begin{abstract}
Finding new ways for the preparation of cross-linked structures is a significant problem in terms of materials for biomedical application, lithium batteries electrolytes, and etc. Within this work we have studied the possibility to utilize hydrosilylation and Piers-Rubinsztajn reactions to obtain cross-linked model phosphazene compounds, containing eugenoxy and guaiacoxy groups. It was shown that Piers-Rubinsztajn reaction cannot be efficiently used to prepare tailored polymermatrix, due to the catalyst deactivation by nitrogen atoms of phosphazene units. A number of crosslinked phosphazene-based materials was obtained with the use of hydrosilylation reaction and their properties were studied by NMR spectroscopy, FTIR, DSC, and TGA. This work showed a perspective for the use of eugenoxy functional groups for the preparation of three-dimensional hybrid phosphazene/siloxane-based materials for various applications.
\end{abstract}

Keywords: phosphazenes, cross-linked, hydrosilylation, Piers-Rubinsztajn reaction, eugenol, siloxanes, model compounds, siloxane-phosphazene.

\section{Introduction}

The rapid development of technological progress demands preparation of novel and effective current sources, which can work in the wide temperature range, possess high charge capacity and rechargeability [1-3]. Among all polymers, used for lithium-ion batteries, polyethyleneoxide (PEO) is one of the most studied, which possesses good ion conductivity due to high flexibility of polymeric chain and its ability for coordination of lithium ions with oxygen atoms [4]. The main disadvantage of this polymer is its high tendency to crystalline phase formation, which in turn leads to a decrease of ion conductivity [5]. On the other hand, phosphazene polymers are good candidates for polyelectrolyte materials since they obtain high flexibility of polymeric chain, high chemical and thermal stability, biocompatibility, fire-proofing and easy backbone derivatization [6-11]. Moreover, phosphazene chain can also contribute to metal cations coordination due to the presence of electron rich nitrogen atoms in the $\mathrm{P}=\mathrm{N}$ structure $[8,9,12]$. The most known efficient phosphazene alternative for PEO is poly(methoxyethoxyethoxy)phosphazene (PMEEP) - fully amorphous polymer $[5,13,14]$. Despite a number of obvious benefits like very low glass transition temperature and 6 oxygen atoms per 1 chain unit, PMEEP possesses quite low dimensional stability. One route to overcome this drawback is cross-linking, which, by the way, can also prevent the formation of lithium dendrites during the charging/discharging processes [15]. Phosphazene cross-linking is traditionally done using $\mathrm{Co}^{60}$ radiation through C-H bonds cleavage, but the process is not well controlled and can yield too hard matrix. At the same time, the formation of rigid tailored network will decrease the segmental motion and as a result ionic conductivity, so the cross-linking units are to be quite flexible. In light of this, functional highly-flexible oligosiloxanes seem to be ideal cross-linkers [16-18]. On the other hand, eugenol fragments are promising functional groups to be introduced into polyphosphazene structure [19-21]. Eugenoxy fragments on phosphorus atoms have two active centers, which can react with hydride-containing siloxanes by different ways: allylic groups through the hydrosilylation and 
methoxy groups through Piers-Rubinsztajn reaction [22]. In this work we have synthesized model compounds hexaeugenoxycyclotriphosphazene and hexaguaiacoxycyclotriphosphazene and studied their ability for cross-linking with hydride terminated oligosiloxanes.

\section{Materials and Methods}

\subsection{Materials}

Eugenol ( $\geq 98 \%$ pure, Acros Organics) was distilled prior to use. Hexachlorocyclotriphosphazene was obtained from Rushim (Moscow, Russia) and purified by vacuum sublimation. Sodium metal and hydride-terminated oligodimethylsiloxane $\mathrm{HSi}\left(\mathrm{CH}_{3}\right)_{2}\left(\mathrm{Si}_{(}\left(\mathrm{CH}_{3}\right)_{2} \mathrm{O}\right)_{6} \mathrm{Si}\left(\mathrm{CH}_{3}\right)_{2} \mathrm{H}$ were purchased from Sigma-Aldrich Corp. (St. Louis, MO, USA) and used as received.

Karstedth catalyst (toluene solution, $\mathrm{C}_{\mathrm{Pt}}=1 \mathrm{mg} / \mathrm{ml}$ ), obtained from Penta-91 (Moscow, Russia), and tris(pentafluorophenyl)borane $\mathrm{B}\left(\mathrm{C}_{6} \mathrm{~F}_{5}\right)_{3}$, purchased from P\&M-invest (Moscow, Russia), were used without any additional purification.

All solvents (dioxane, toluene) were purified according to the known methods [23] and were used as freshly distilled. The water content was controlled in ppm by Metrohm 899 Coulometer. All reactions were carried out under inert atmosphere of dry nitrogen using standard Schlenk techniquies.

\subsection{Characterization methods}

${ }^{31} \mathrm{P}$ and ${ }^{1} \mathrm{H}$ NMR spectra were recorded by Bruker CXP-200 spectrometer operating at frequencies 81 and $200 \mathrm{MHz}$, respectively. The spectra were recorded in $\mathrm{CDCl}_{3}$ and reported in parts per million $(\delta)$ relative to residual solvent signal for ${ }^{1} \mathrm{H}(7.26 \mathrm{ppm})$ spectra, and $85 \% \mathrm{H}_{3} \mathrm{PO}_{4}(0.0 \mathrm{ppm})$ as external standard for ${ }^{31} \mathrm{P}$ NMR spectra.

FTIR spectra were measured with the use of Nicolet iS50 (Thermo Fisher Scientific) spectrometer in ATR mode with diamond crystal.

Differential scanning calorimetry (DSC) was performed on a Netzsch DSC 204 F1 Phoenix instrument (Netzsch, Selb, Germany) in a nitrogen atmosphere $(20 \mathrm{~mL} / \mathrm{min})$ at a heating rate of 20 $\mathrm{deg} / \mathrm{min}$ on samples weighing $~ 5-8 \mathrm{mg}$.

Thermal gravimetric analysis (TGA) was performed on Derivatograph-1500Q at a heating rate of $20 \mathrm{deg} / \mathrm{min}$ in an air atmosphere.

\subsection{Synthesis of cyclic phosphazene $P_{3} N_{3} G u a_{6}$ or $P_{3} N_{3} E u g_{6}$.}

$15.70 \mathrm{~g}(0.1264 \mathrm{~mol})$ of guaiacol, in case of $\mathrm{P}_{3} \mathrm{~N}_{3} \mathrm{Gua}$, or $20.76 \mathrm{~g}(0.1264 \mathrm{~mol})$ of eugenol, in case of $\mathrm{P}_{3} \mathrm{~N}_{3} \mathrm{Eug}_{6}$, and $150 \mathrm{~mL}$ of dioxane were charged into the three-necked flask, equipped with a magnetic stirrer and reflux condenser. Then $2.64 \mathrm{~g}(0.1149 \mathrm{~mol})$ of sodium were added by thin cutted plates and the mixture was stirred at $70{ }^{\circ} \mathrm{C}$ until the full dissolution of sodium. Then a solution of $5.00 \mathrm{~g}(0.0144 \mathrm{~mol})$ of hexachlorocyclotriphosphazene in $50 \mathrm{~mL}$ of dioxane was added dropwise and the reaction mixture was stirred at $70{ }^{\circ} \mathrm{C}$ for $24 \mathrm{~h}$, afterwards poured into the excess of water. The formed precipitate was dissolved in chloroform, washed with water and the resulted solution was dried over $\mathrm{Na}_{2} \mathrm{SO}_{4}$. Then chloroform was rotary evaporated, and the product was dissolved in mixture of $30 \mathrm{~mL}$ of dichloromethane and $30 \mathrm{~mL}$ of ethanol. After slow dichloromethane evaporation, a yellow crystalline product was formed, which was then dried at $60^{\circ} \mathrm{C}$ under vacuum for $4 \mathrm{~h}$. Yields: $9.82 \mathrm{~g}(78.20 \%)$ of $\mathrm{P}_{3} \mathrm{~N}_{3} \mathrm{Gua}_{6}$ and $10.97 \mathrm{~g}(68.53 \%)$ of $\mathrm{P}_{3} \mathrm{~N}_{3} \mathrm{Eug}_{6}$.

\subsection{Preparation of hybrid cross-linked phosphzene-siloxane materials}

$0.2 \mathrm{~g}$ of eugenoxy-substituted phosphazene trimer were dissolved in toluene. Then subsequently a catalyst $\left(\mathrm{B}_{(} \mathrm{C}_{6} \mathrm{~F}_{5}\right) 3$ in toluene $(40 \mathrm{mg} / \mathrm{ml})$ or Karstedth catalyst solution in toluene $\left(\mathrm{C}_{\mathrm{Pt}}=1 \mathrm{mg} / \mathrm{ml}\right)$ and hydride terminated oligodimethylsiloxane $\mathrm{HSi}\left(\mathrm{CH}_{3}\right)_{2}\left(\mathrm{Si}_{2}\left(\mathrm{CH}_{3}\right)_{2} \mathrm{O}\right)_{6} \mathrm{Si}\left(\mathrm{CH}_{3}\right)_{2} \mathrm{H}$ were added. Then the mixture was cured for $2 \mathrm{~h}$ at $80{ }^{\circ} \mathrm{C}$. The more detailed data about catalyst, solvent and siloxane amounts are shown in Table 2. 


\section{Results and discussion}

At first a cyclotriphosphazenes, containing eugenoxy- or guaiacoxy- groups were synthesized (Scheme 1). The reaction between haxachlorocyclotriphosphazene and sodium salt of eugenol or guaiacol was carried out in a medium of dioxane as a solvent at $70^{\circ} \mathrm{C}$.
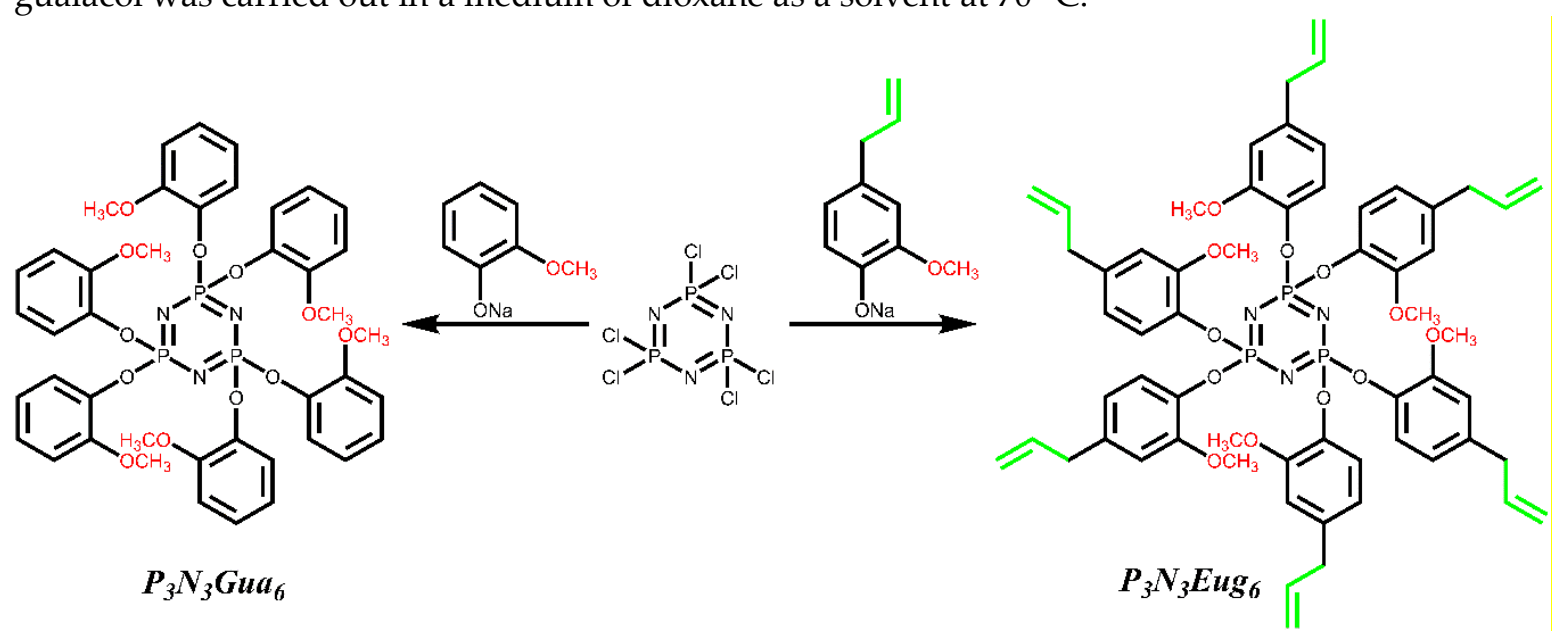

Scheme 1. Synthesis of $\mathrm{P}_{3} \mathrm{~N}_{3} \mathrm{Gua} 6$ and $\mathrm{P}_{3} \mathrm{~N}_{3}$ Eug6.

The structure of the product was confirmed by NMR spectroscopy. In ${ }^{1} \mathrm{H}$ spectrum signals at 6.4-7 and $3.7 \mathrm{ppm}$ are attributed to protons of aromatic and methoxy groups, respectively. Signals of allyl group at 3.3, 5.0 and $5.9 \mathrm{ppm}$ can be observed as well, which fully corresponds to the literature data [21]. In ${ }^{31} \mathrm{P}$ spectrum signals at 9.0 and $8.7 \mathrm{ppm}$ stand for $\mathrm{P}_{3} \mathrm{~N}_{3} \mathrm{Gua}_{6}$ and $\mathrm{P}_{3} \mathrm{~N}_{3} \mathrm{Eug}_{6}$, respectively. Other peaks in ${ }^{31} \mathrm{P}$ spectrum, triplet at $21.9 \mathrm{ppm}$ and douplet at $6.6 \mathrm{ppm}$, are attributed to the incompletely substituted cyclotriphosphazene compound $\mathrm{P}_{3} \mathrm{~N}_{3} \mathrm{ClEug} 5$.
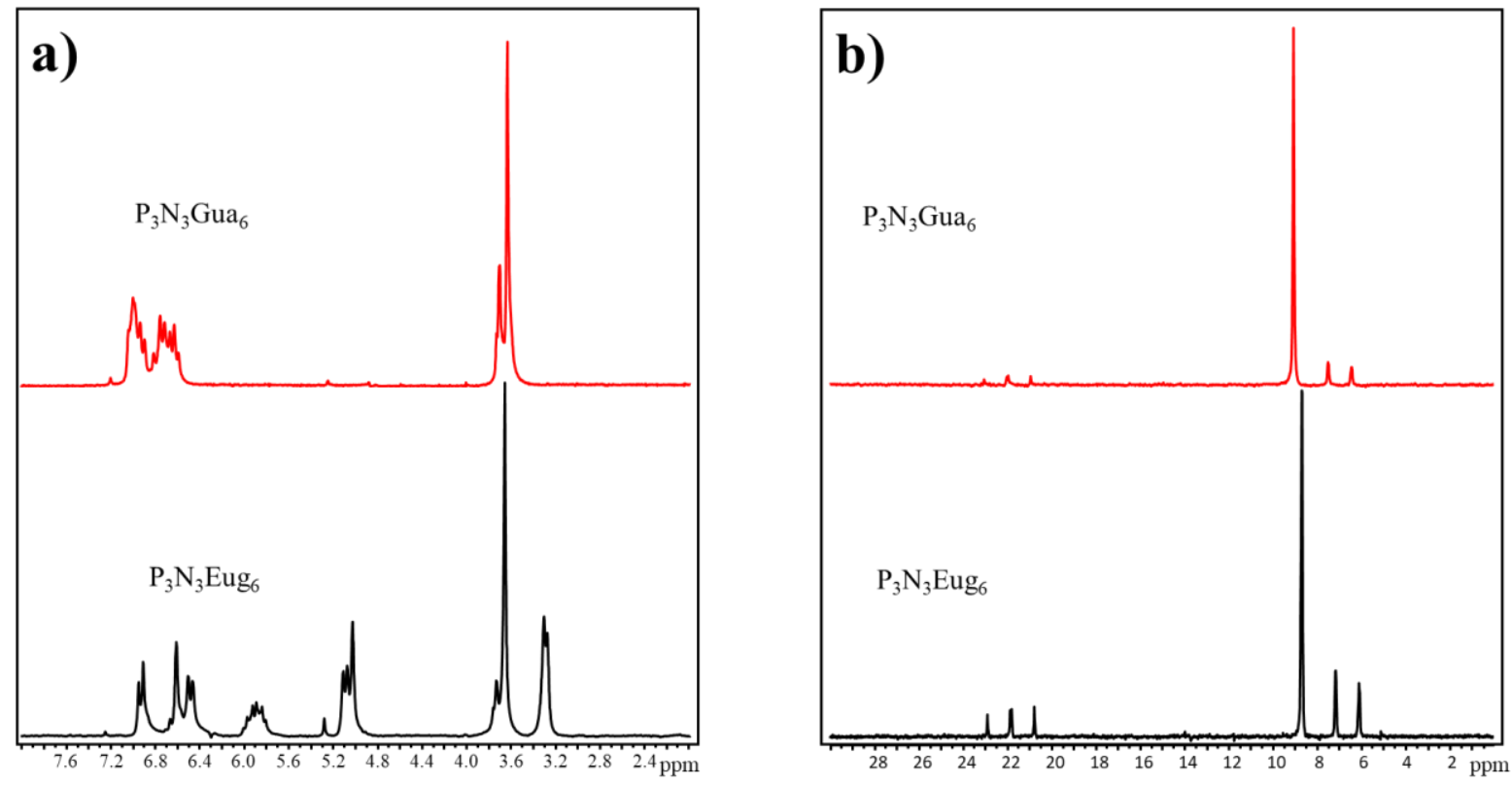

Figure 1. ${ }^{1} \mathrm{H}$ (a) and ${ }^{31} \mathrm{P}(\mathrm{b}) \mathrm{NMR}$ spectra of $\mathrm{P}_{3} \mathrm{~N}_{3} \mathrm{Gua} 6$ and $\mathrm{P}_{3} \mathrm{~N}_{3} \mathrm{Eug}_{6}$.

The prepared fully derivatized products were planned to undergo the reactions with hydrosilyl groups by Piers-Rubinsztajn and hydrosilylation processes. It should be noted here that the most suitable solvents for carrying out these reactions are non-polar toluene and hexane. However, in these media, the hexaguaiacoxycyclophopshazene turned out to be almost completely insoluble, and therefore attempts were made to carry out the reaction in block, as well as in polar THF. Unfortunately, both variants did not give positive results: in the absence of a solvent, phosphazene turned out to be incompatible with oligosiloxane, and in polar THF, despite the satisfactory solubility 
of the product, hydrosilylation and Piers-Rubinsztajn reaction did not proceed. Thus, further studies were carried out only with a hexaeugenoxy- derivative (Scheme 2).

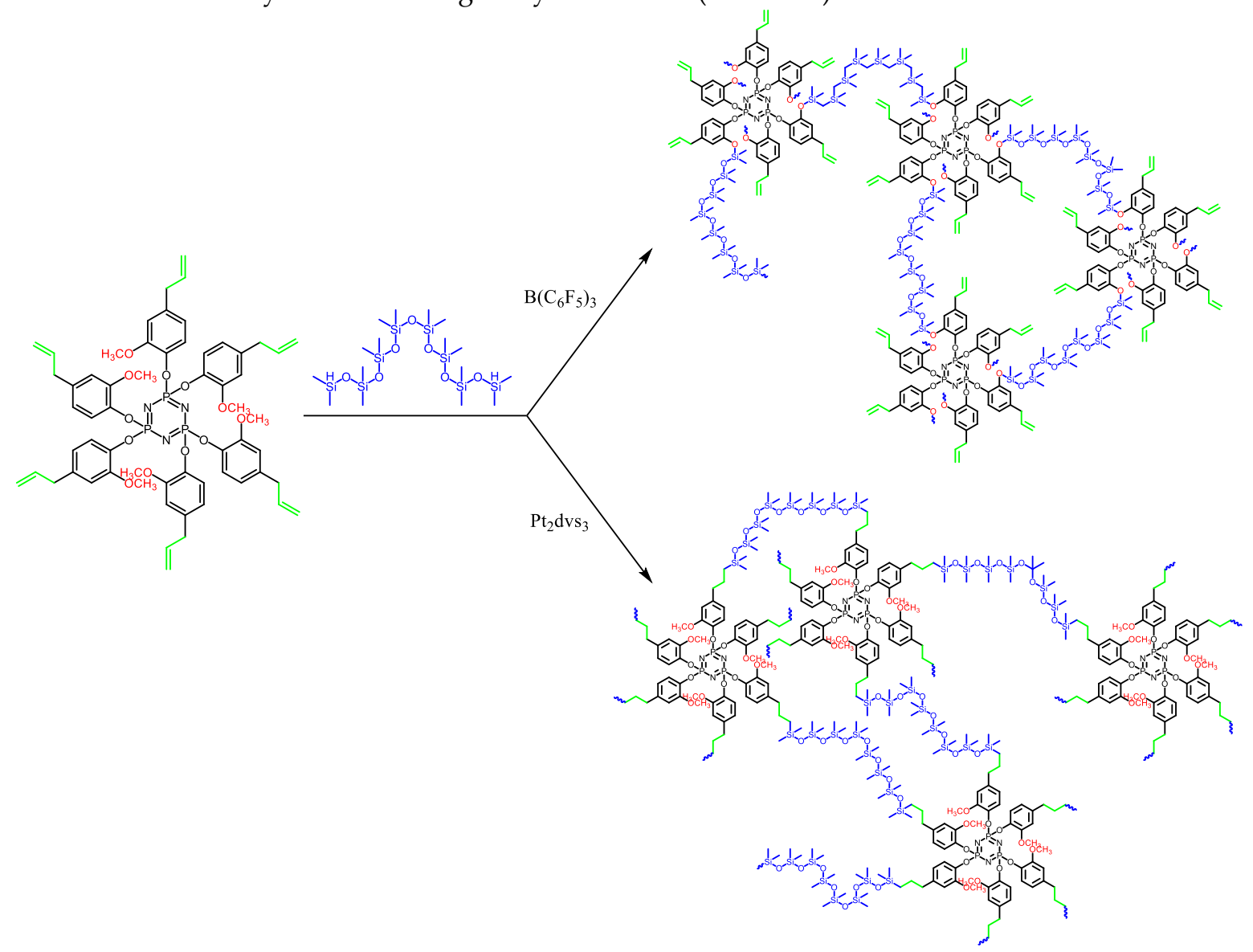

Scheme 2. Cross-linking of $\mathrm{P}_{3} \mathrm{~N}_{3} \mathrm{Eug}_{6}$ by means of hydrosilylation and Piers-Rubinsztajn reaction.

On the first step, a model hexaeugenoxycyclotriphosphazene was used as a model compound for finding out the optimal reaction conditions during the interaction with hydrogen-terminated oligodimethylsiloxane. For Piers-Rubinsztajn reaction it was found that no reaction takes place no matter what condition are supported. This can be explained by the deactivation of Lewis acid catalyst with nitrogen atoms of phosphazene units, exhibiting Lewis base properties [24], on the one hand, or due to the sterical hindrances for methoxy groups, on the other hand. This problem will be additionally studied in details in future.

On the next step, hydrosilylation was used. To study the reaction conditions influence on the final materials properties, we varied such parameters as catalyst amount, concentration of initial $\mathrm{P}_{3} \mathrm{~N}_{3} \mathrm{Eug}_{6}$ in toluene and molar ratio between $\mathrm{P}_{3} \mathrm{~N}_{3} \mathrm{Eug}_{6}$ and hydride terminated oligosiloxane (Table 2).

Table 2. Loadings for the preparation of cross-linked materials based on $\mathrm{P}_{3} \mathrm{~N}_{3} \mathrm{Eug}_{6}$, obtained via hydrosilylation reactions.

\begin{tabular}{|c|c|c|c|c|c|}
\hline $\begin{array}{c}\text { \# of } \\
\text { Sample }\end{array}$ & $\begin{array}{l}\text { Molar ratio } \\
\mathrm{P}_{3} \mathrm{~N}_{3} \text { Eug }_{6} \text { : } \\
\text { siloxane }\end{array}$ & $\begin{array}{c}\text { Catalyst } \\
\text { amount, } \\
\text { mol. } \%\end{array}$ & $\begin{array}{c}\text { Concentration } \\
\text { of } \mathrm{P}_{3} \mathrm{~N}_{3} \mathrm{Eug}_{6} \text { in } \\
\text { toluene, } \\
\mathrm{mg} / \mathrm{mL}\end{array}$ & Result & $\mathrm{T}_{\mathrm{g},}{ }^{\circ} \mathrm{C}$ \\
\hline 1 & $1: 3$ & 0.0015 & 50 & Yellowish sticky film* & 5.3 \\
\hline 2 & $-/ /-$ & 0.003 & 50 & Yellowish hard film & -5.1 \\
\hline 3 & $-/ /$ & 0.006 & 50 & Yellowish hard film & -9.2 \\
\hline 4 & $-/ /-$ & 0.003 & 25 & Yellowish sticky film & -13.7 \\
\hline 5 & $-/ /-$ & $-/ /-$ & 100 & Yellowish hard film & -7.5 \\
\hline
\end{tabular}




\begin{tabular}{llllll}
\hline 6 & $1: 2$ & $-/ /-$ & $-/ /-$ & Yellowish hard film & -5.3 \\
7 & $1: 1$ & $-/ /-$ & $-/ /-$ & Yellowish soluble resin & -4.8 \\
\hline
\end{tabular}

${ }^{*}$ No cross-linking was observed after $2 \mathrm{~h}$ of curing process at $80{ }^{\circ} \mathrm{C}$. The cross-linked film was formed after $5 \mathrm{~h}$ of curing process.

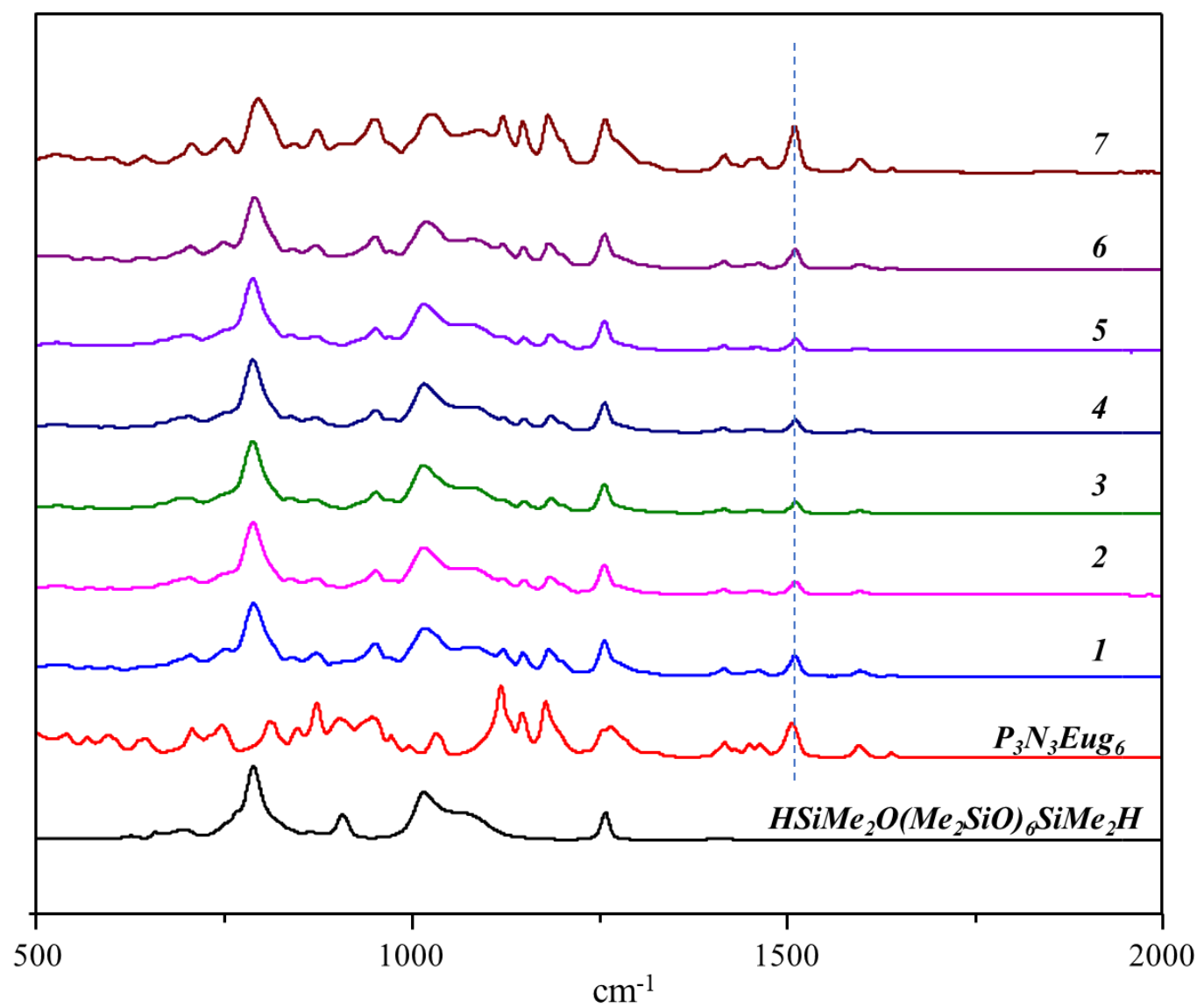

Figure 2. FTIR spectra of initial monomers and cross-linked polymers.

FTIR spectra (Figure 2) show that change of these conditions influences on cross-linking degree, which can be evaluated by changing of the intensity of peak at $1510 \mathrm{~cm}^{-1}$, attributed to double bonds of allyl groups. So, in a row of polymers from 1 to 3 the intensity of this peak decreases along with the increase of catalyst amount used. For polymers 7,6 and 5 the intensity decreases as the molar ratio $\mathrm{P}_{3} \mathrm{~N}_{3} \mathrm{Eug}_{6}$ : siloxane changes from 1:1 to 1:3.

Thermal properties were studied based on the polymer 5 sample (Figure 3). It was shown that the polymer starts to decompose at $390{ }^{\circ} \mathrm{C}$ with char yield of $40 \%$ at $800{ }^{\circ} \mathrm{C}$. An initial small weight loss at temperatures less than $390^{\circ} \mathrm{C}$ is attributed to removal of residual toluene. The higher char yield and degradation temperature in comparison with the initial monomer $\mathrm{P}_{3} \mathrm{~N}_{3} \mathrm{Eug}_{6}(10 \%$ and 330 ${ }^{\circ} \mathrm{C}$ ) is explained by the cross-linked structure of the investigated polymer. 


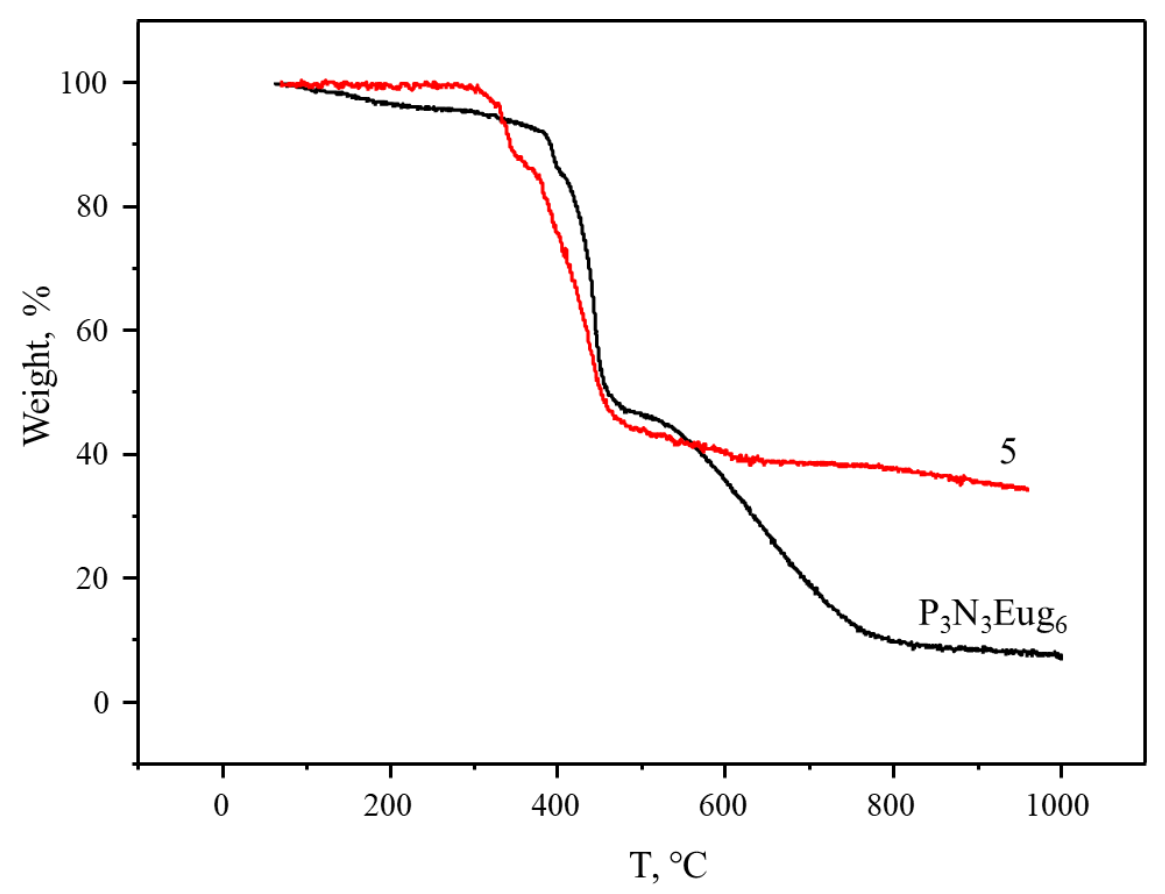

Figure 3. TGA curves of $\mathrm{P}_{3} \mathrm{~N}_{3} \mathrm{Eug}_{6}$ and polymer 5.

According with data from Table 2, the glass transition temperature increases with the decrease of cross-linking degree. For example, polymer 7 with lower cross-linking degree has higher $\mathrm{T}_{\mathrm{g}}(-4.8$ $\left.{ }^{\circ} \mathrm{C}\right)$ in comparison with polymer $5\left(-7.5^{\circ} \mathrm{C}\right)$ and $6\left(-5.3^{\circ} \mathrm{C}\right)$. Same situation is also observed for polymers 1, 2 and 3. Such phenomenon probably can be explained by two reasons. First, the more cross-linked polymers can contain higher amount of entrapped residual toluene, which can serve as a plasticizing agent. Second, in highly cross-linked polymers the segmental mobility is provided mostly by flexible siloxane units. When the cross-linking degree decreases, the hard cyclophosphazene units can also lose segmental mobility to some extent and so the glass transition temperature will increase.

\section{Conclusions}

In conclusion, we have studied a possibility for cross-linking of phosphazene compounds, having eugenoxy- and guaiacoxy- groups. It was shown, that Piers-Rubinsztajn reaction is not the appropriate method due to the deactivation of Lewis acid catalyst with the nitrogen atoms of phosphazene units. On the other hand, the hydrosilylation reaction can be used for the preparation of hybrid phosphazene/siloxane-based polymeric materials with fine-tunable properties. These results open a new perspective in preparation of novel functional phosphazene-based cross-linked structures for such applications as lithium batteries, dentistry, biomedical application, elastomers etc.

Author Contributions: Conceptualization, M.V. Gorlov and M.A. Soldatov; methodology, A.I. Chernyshova A.S. Esin, and E.A. Karpova; writing - original draft preparation, M.A. Soldatov; writing - review and editing, M.V. Gorlov; supervision, I.S. Sirotin and M.V. Gorlov; project administration, V.V. Kireev. All authors have read and agreed to the published version of the manuscript.

Funding: This research was funded by Mendeleev University of Chemical Technology of Russia. Project number 2020-035.

Conflicts of Interest: The authors declare no conflict of interest.

\section{References}

1. Manthiram, A.; Fu, Y.; Chung, S.-H.; Zu, C.; Su, Y.-S. Rechargeable Lithium-Sulfur Batteries. Chem. Rev. 2016, 114, 11751-11787. 
2. Muench, S.; Wild, A.; Friebe, C.; Häupler, B.; Janoschka, T.; Schubert, U.S. Polymer-Based Organic Batteries. Chem. Rev. 2016, 116, 9438-9484.

3. Zheng, Y.; Yao, Y.; Ou, J.; Li, M.; Luo, D.; Dou, H.; Li, Z.; Amine, K.; Yu, A.; Chen, Z. A review of composite solid-state electrolytes for lithium batteries: fundamentals, key materials and advanced structures. Chem. Soc. Rev. 2020, 49, 8790-8839.

4. Xue, Z.; He, D.; Xie, X. Poly(ethylene oxide)-based electrolytes for lithium-ion batteries. J. Mater. Chem. A 2015, 3, 19218-19253.

5. Xu, K. Nonaqueous Liquid Electrolytes for Lithium-Based Rechargeable Batteries. Chem. Rev. 2004, 104, 4303-4417.

6. Allcock, H.R.; Chen, C. Polyphosphazenes: Phosphorus in Inorganic-Organic Polymers. J. Org. Chem. 2020, 85, 14286-14297.

7. Gorlov, M.V.; Bredov, N.S.; Esin, A.S.; Kireev, V.V. A direct synthesis of $\mathrm{Cl3P}=\mathrm{NSiMe} 3$ from $\mathrm{PCl} 5$ and hexamethyldisilazane. J. Organomet. Chem. 2016, 818, 82-84.

8. Bredov, N.S.; Gorlov, M.V.; Esin, A.S.; Bykovskaya, A.A.; Kireev, V.V.; Sinegribova, O.A.; Ryabochenko, M.D. Linear 2-ethylhexyl imidophosphoric esters as effective rare-earth element extractants. Appl. Sci. 2020, 10(4), 1229.

9. Soldatov, M.; Liu, H. A POSS-Phosphazene Based Porous Material for Adsorption of Metal Ions from Water. Chem. Asian J. 2019, 14(23), 4345-4351.

10. Onuchin, D.V.; Sirotin, I.S.; Sarychev, I.A.; Bornosuz, N.V.; Kireev, V.V.; Gorbunova, I.Y.; Gorbatkina, Y.A. Physicochemical Properties of Epoxy Composites Modified with Epoxyphosphazene. Polym. Sci. Ser. B 2019, 61(3), 286-293.

11. Sirotin, I.S.; Sarychev, I.A.; Vorobyeva, V.V.; Kuzmich, A.A.; Bornosuz, N.V.; Onuchin, D.V.; Gorbunova, I.Yu.; Kireev, V.V. Synthesis of Phosphazene-Containing, Bisphenol A-Based Benzoxazines and Properties of Corresponding Polybenzoxazines. Polymers 2020, 12(6), 1225

12. van Wüllen, L.; Köster, T.K.-J.; Wiemhöfer, H.-D.; Kaskhedikar N. Local Cation Coordination Motifs in Polyphosphazene Based Composite Electrolytes. Chem. Mater. 2008, 20, 7399-7407.

13. Blonsky, P.M.; Shriver, D.F.; Austin, P.; Allcock, H.R. Polyphosphazene Solid Electrolytes. J. Am. Chem. Soc. 1984, 106, 6854-6855.

14. Allcock, H.R.; Ravikiran, R.; O'Connor, S.J.M. Effect of Oligo(ethyleneoxy)cyclotriphosphazenes, Tetraglyme, and Other Small Molecules on the Ionic Conductivity of the Poly[bis(methoxyethoxyethoxy)phosphazene] (MEEP)/Lithium Triflate System. Macromolecules 1997, 30, 3184-3190.

15. Stalin, S.; Johnson, H.E.N.; Biswal, P.Vu.D.; Zhao, Q.; Yin, J.; Abel, B.A.; Deng, Y.; Coates, G.W.; Archer, L.A. Achieving Uniform Lithium Electrodeposition in Cross-Linked Poly(ethylene oxide) Networks: "Soft" Polymers Prevent Metal Dendrite Proliferation. Macromolecules 2020, 53, 5445.

16. Wang, G.-J.N.; Shaw, L.; Xu, J.; Kurosawa, T.; Schroeder, B.C.; Oh, J.Y.; Benight, S.J.; Bao Z. Inducing Elasticity through Oligo-Siloxane Crosslinks for Intrinsically Stretchable Semiconducting Polymers. Adv. Funct. Mater. 2016, 26, 7254-7262.

17. Shaik, A.; Narayan, R.; Raju, K.V.S.N. Synthesis and properties of siloxane-crosslinked polyurethaneurea/silica hybrid films from castor oil. J. Coat. Technol. Res. 2014, 11 (3) 397-407.

18. Kolel-Veetil, M.K.; Keller, T.M. Formation of Elastomeric Network Polymers from Ambient Heterogeneous Hydrosilations of Carboranylenesiloxane and Branched Siloxane Monomers. J. Polym. Sci. A Polym. Chem. 2006, 44, 147-155.

19. Kireev, V.V.; Bilichenko, Yu.V.; Borisov, R.S.; Sirotin, I.S.; Filatov, S.N. Laser Mass Spectrometry Analysis of the Formation of Phosphazene-Containing Epoxy Oligomers. Polym. Sci. Ser. B 2018, 60, 243-262.

20. Sirotin, I.S.; Bilichenko, Yu.V.; Solodukhin, A.N.; Kireev, V.V.; Buzin, M.I.; Borisov, R.S. Eugenol Derivatives of Higher Chlorocyclophosphazenes and Related Epoxy Oligomers. Polym. Sci. Ser. B 2013, 55, 241-251.

21. Kireev, V.V.; Bredov, N.S.; Prudskov, B.M.; Mu, J.; Borisov, R.S.; I. B. Sokol'skaya, I.B.; Chuev, V.P. Oligo and Polysiloxanephosphazenes Based on Eugenol Cyclotriphosphazene Derivatives. Polym. Scie. Ser. B 2011, 53, 64-67

22. Laengert, S.E.; Schneider, A.F.; Lovinger, E.; Chen, Y.; Brook, M.A. Sequential Functionalization of a Natural Crosslinker Leads to Designer Silicone Networks. Chem. Asian J. 2017, 12, 1208-1212.

23. Armarego, W.L.F.; Chai, C.L.L. Purification of Laboratory Chemicals. 6th ed.; Elsevier: Burlington, USA, 2009; 760 p. 
24. John B. Grande, J.B.; Thompson, D.B.; Gonzaga F.; Brook, M.A. Testing the functional tolerance of the PiersRubinsztajn reaction: a new strategy for functional silicones. Chem. Commun. 2010, 46, 4988-4990. 